\title{
A MODIFIED SPATIAL FUZZY C-MEANS CLUSTERING ALGORITHM FOR DETECTING GLAUCOMAIN RETINAL FUNDUS IMAGES
}

\author{
S.J. Grace Shoba and A. Brintha Therese \\ School of Electronics Engineering, VIT University, Chennai, Tamil Nadu, India
}

Received 2013-10-12; Received 2014-02-25; Accepted 2014-03-05

\begin{abstract}
Glaucoma is a disease which affects the eye and causes blindness. It is an ophthalmologist disease characterized by an increase in Intraocular Pressure (IOP). The glaucoma usually affects the optic disc on the retina which increases the cup size. There are various parameters to identify and diagnose glaucoma. The clustering technique is introduced to detect the glaucoma from the optic disc and cup in the retinal fundus images. Fuzzy C Means (FCM) Clustering is used for clustering the data in which the data points are clustered with different membership degree. But it does not fully utilize the spatial information in the image. The Modified Spatial Fuzzy C-Means clustering with spatial rotation has been proposed to detect glaucoma in retinal fundus images. The first and foremost step is preprocessing operation, in which the optic cup and disk of the input image is being rotated. Initially the optic disk is rotated in some angle and the distance between the data points are measured and a cluster is formed based on the centroid. The centroid and data point along with the cluster can be identified in each step then the common set of points is clustered together. This process continues until no more centroid is found. The cluster with more data points that do not match with the original image is considered as the retinal image with glaucoma disease. In future, this algorithm can be extended to larger clinical databases in order to identify the glaucoma at the maximum level.
\end{abstract}

Keywords: Glaucoma, Spatial Fuzzy C Means Clustering, Spatial Information, Fundus Image

\section{INTRODUCTION}

Normally the human eye sends the light signal to the visual cortex which is located at the back of the brain. It contains the iris, the lens, the pupil, the retina and the optic nerve. Retina is the innermost coat of the eye which carries the visual impulse to the optic nerve. The optic disc is located at the back of the eye with the bundle of nerve fibers that carry visual information to the brain. Glaucoma is an eye disease in which the fluid pressure within the eye rises and leads to the loss of human vision. There are two types of glaucoma namely open angle and closed angle. In open angle glaucoma the patient may not feel any symptoms, whereas closed angle glaucoma the patients may get the pain and vision loss. The treatment of glaucoma is either by the Corresponding Author: S.J. Grace Shoba, School of Electronics Engineering, VIT University, Chennai, Tamil Nadu, India

application of eye drop or surgery, it will not suitable for all patients because, they cannot tolerate the pain (Narasimhan and Vijayarekha, 2011; Anjana et al., 2012). Glaucoma is not a curable disease which losses the vision of the eye and cannot be restored. There are various factors to be considered for diagnosing the glaucoma such as inner eye pressure, the shape and color of the optic nerve, complete field of vision and angle in the eye where the iris meets the cornea, thickness of the cornea and so on. A remarkable amount of research has been reported on the early detection of glaucoma. The Principal Component Analysis (PCA) is used to localize and shape the optic disk by using the model called modified Active Shape Model (ASM) for glaucoma detection. The fundus image features are extracted with the success rates as 
99, 94 and $100 \%$ for disk localization, disk boundary detection and fovea localization re-spectively. These models can be improved in larger data source and also used for clinical purposes (Mary and Marri, 2012). A novel framework in automatic detection of optic disc and macula in retinal fundus images are represented by a weighted complete graph. The nodes in the graph are pruned i.e., the worst vertices are removed from the graph which is based on the predefined geometric constraints (Kovacs et al., 2010). A fast segmentation processes has been proposed in K-means based clustering and in hierarchical K-means based clustering with spatial constraints at each level. These processes are carried out to get an efficient clustering in the segmented images (Raed and Ashour, 2013). An automatic and simultaneous detection method is used to detect and predict the structure of optic disc which gives better result when compared to the other detection methods. The features are extracted from the fundus image; finds out the feature set and calculates the distance variable. The K-NN classifier is used to extract and train the feature set in order to achieve the performance (Xiayu, 2010). The reliable fovea and optic disk detection method is used to detect the center and radius of the optic disk and to identify the location of the fovea. The shape detection is followed by the Hough transform which has been carried out for finding the optic disk (Pinao, 2011). An automatic, precise, 3-D optic nerve head reconstruction method has been proposed for performing a sparse-image registration and dense-depth recovery. The correlation method and feature based method are also proposed for handling a noise and accuracy in the fundus images respectively. The sub pixel matching is used to match the sparse points in low resolution images (Lee et al, 2013). An automatic method for segmenting the ARMD in retinal fundus images with a simple inverse segmentation method has been proposed to utilize the homogeneity of healthy areas in the macula. This method starts with simple region growing method then the blood vessels are extracted and classified as healthy regions. The proposed segmentation method achieves the accuracy rate as $90 \%$ (Kose et al., 2012). A new adaptive method has been proposed for the automatic segmentation of the optic disk in digital color fundus images. There are two data-bases namely DRIVE and DIARETDB1 were used to locate the optic disk with success rate 100 and $97.75 \%$ respectively (Welfer et al., 2010). Many image segmentation methods are available for reliable processing of any medical images. It can be classified into edge based image segmentation and region based image segmentation. Hierarchical clustering is a region based image segmentation technique which provides simple and reliable clustering process, but it may increases the computational time. So, it is not suitable for large images. The K-means clustering algorithm is a partition clustering method in which the number of cluster fixed in advance, so it is a simple and fastest method. This method has suffered in an issue over the clustering process because the cluster size is fixed in advanced with different initial parameters. The region growing segmentation method gives the good segmentation result in order to specify the region with correct parameters. It takes too much time to complete the clustering process, so it is expensive. The region splitting and merging method will split the images until the correct resolution is reached. It is not suitable for more number of images processed at the same time. Watershed is the edge based image segmentation method provides a large number of segmented images with high reliability which also suffers in over segmentation (Yahya et al., 2013). Fuzzy C means (FCM) is a data clustering technique in which a data set is grouped into ' $n$ ' clusters with every data point in the dataset which belongs to every cluster to a certain degree. A conventional FCM algorithm does not incorporate the spatial information which makes it sensitive to noise and other image artifacts whereas Spatial Fuzzy C means clustering algorithm incorporates the spatial information into the membership function for clustering.

The Modified Spatial Fuzzy C-Means clustering method is used to detect glaucoma which is present in the retina with various spatial coordinates. The rest of the paper organized as follows, section 1 presents the overview of glaucoma and various clustering algorithms. Section 2 presents the proposed methodology of Modified Spatial Fuzzy C Means clustering. Section 3 presents the histogram analysis of glaucoma detection. Section 4 presents the algorithm for the Modified Spatial Fuzzy C Means clustering model. Section 5 presents the comparison of various algorithms. Section 6 presents the spatial analysis of glaucoma detection. Finally, section 7 presents the conclusion and future work.

\section{PROPOSED METHODOLOGY}

Figure 1 shows that the overall methodology of the proposed system. The image is acquired from the image 
sources. This acquired image will be converted into gray scale image in order to perform the image analysis in more efficient manner. The fuzzy parameters are set based on various criteria such as number of clusters, number of iteration and image dimension. The dimension of the input image is checked because the Modified Spatial Fuzzy CMeans clustering is implemented on 2D images. The input image is rotated in various angles to identify the perfect centroid over various data sets. The advantage of spatial rotation is to find out the similarity measures for detecting glaucoma in the retina images. The fuzzy parameters such as membership function, objective function and centroid are used to find a cluster in an efficient way to achieve the high clustering rate. The distance of the data sets are identified based on the fuzzy parameters i.e., the distance measure over the data points are calculated. The clusters are formed based on the spatial fuzzy parameters and also compared with normal cup with same angle of rotation. If two cluster data over spatial fuzzy are same then the eye is not affected by the glaucoma otherwise the eye is affected by the glaucoma.

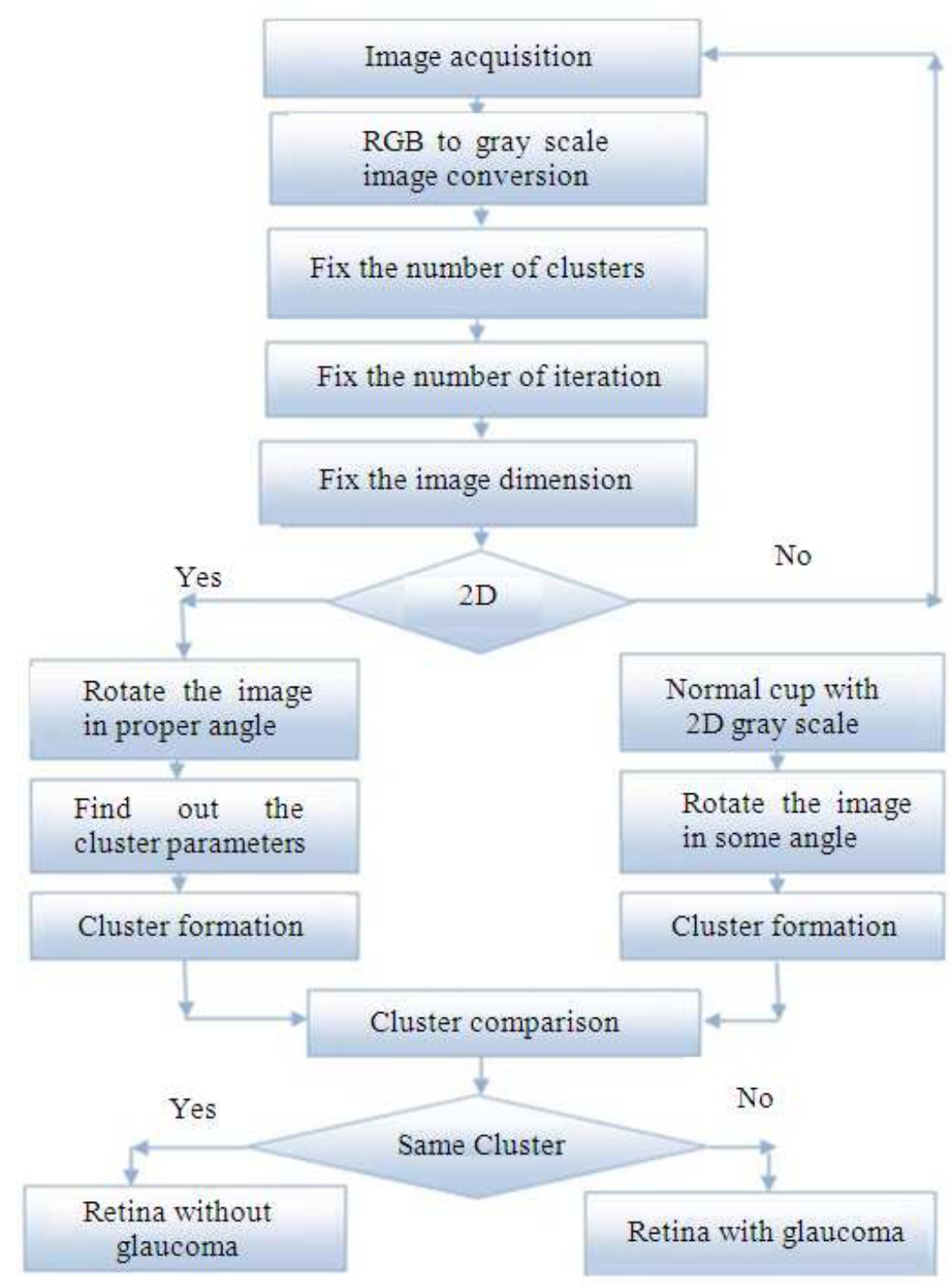

Fig. 1. Flow diagram of the proposed method 

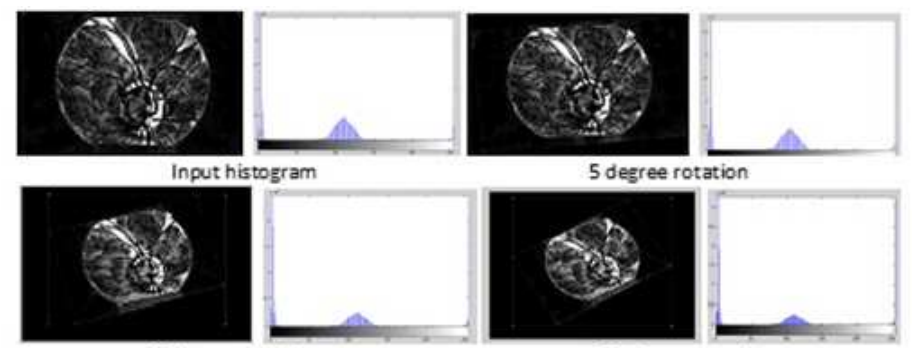

5 degree rotation

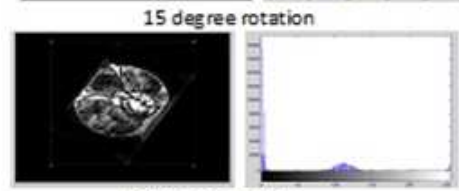

60 degree rotation

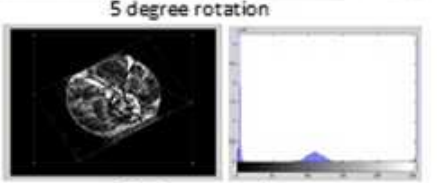

30 degree rotation

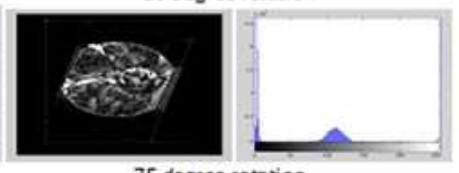

75 degree rotation
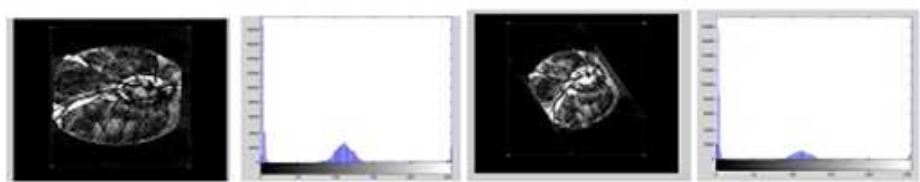

90 degree rotation

120 degree rotation

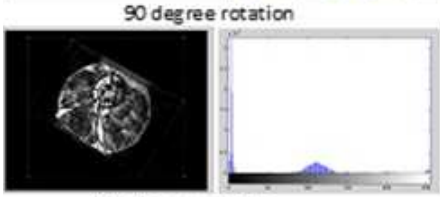

150 degree rotation

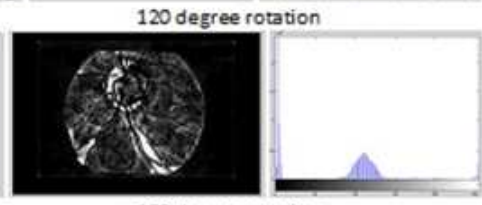

180 degree rotation

Fig. 2. Spatial histogram analysis for input image with various rotations

\section{HISTOGRAM ANALYSIS OF GLAUCOMA DETECTION}

The histogram of the input images are analysed based on various rotations. There are various clusters formed with several angles of rotation. If there is an occurrence of an angle difference then the distance from the centroid also differs ,so the histogram provides the complete analysis of spatial parameters. Figure 2 shows the complete histogram analysis of image with various rotations.

\section{ALGORITHM FOR THE PROPOSED METHODOLOGY}

Algorithm Modified Spatial Fuzzy C Means clustering ()

begin

Determine the Image as IM;

Determine the gray scale images as IG;

Determine the number of clusters as NC;

Determine the iteration as ITR;

Determine the maximum Number of iteration as MAX-ITR;
Determine the number of dimension as DIM; Initialize the fuzzy parameters as FUZZY; Image Rotation as IMGROT;

Image rotation as new cluster and old cluster as NCL

While MAX_ITR not reached true do and OCL respectively;

L2: For each img $\in$ IM do

Convert to the grayscale image equivalent of image

L1: For each gray_image $\in$ IG do if (DIM ! = 2) then error "select the suitable image with DIM" else

Rotate the image with IMGROT;

for each imgrot $\in$ IMGROT and FUZZY do if $(\mathrm{ITR}==1)$ then find out the centroid;

calculate the distance between centriod and data;

maintain a distances as DIST;

find out the objective function by

$\sum\left(\sum((\right.$ dist $\in$ DIST $) 2 *$ MF $\in$ FUZZY $\left.)\right) *$ imgrot;

Cluster formation based on the image with rotation 


\author{
NCL; \\ else \\ for each data and center from ncl $\in$ NCL \\ do \\ for each data and center from ocl $\in$ OCL \\ do \\ find out the membership function for ocl \\ if $((\mathrm{MF} \in \mathrm{OCL})$ and $(\mathrm{MF} \in \mathrm{NCL}))==\operatorname{abs}(\mathrm{MF} \in$ \\ and ncl; \\ form a cluster with fuzzy parameters. \\ FUZZY)) then \\ plot the data along with spatial parameters; \\ goto L1; \\ else \\ goto L2; \\ end \\ end; \\ end; \\ end; \\ end \\ end: Modified Spatial Fuzzy C Means clustering
}

\section{COMPARISION OF VARIOUS CLUSTRING ALGORITHMS}

There are various methods applied over medical image analysis and segmentation. Threshold approach is a method in which the images are segmented, based on the intensities of the image and determine the threshold. The image intensities are classified into two classes which are not suitable for image analysis and segmentation with spatial characterstics. A supervised Learning method is also known as classifiers, requires training data in which the images are segmented by the human. Markov Random Field (MRF) is a statistical model of image segmentation leads a problem while selecting and controlling the parameter, used in spatial interaction. The clustering is a method used to classify the data and also it is used to analyse the image and retrieval of the image without any training set, because clustering follows an unsupervised learning method.

There are three methods commonly used to cluster the data namely K-means, Fuzzy C-Means algorithm and Expectation-Maximization (EM) algorithm. K-means algorithm is a hard segmentation method which clusters a pixel of an image in exactly one class, but it is not suitable for multiple clusters i.e., multiple classes. The Fuzzy C-Means clustering is a soft segmentation method allows one piece of data available in more than one cluster because of different degree of membership. The main drawback of this algorithm degrades the performance due to the noise in images. Fuzzy K-CMeans algorithm (Funmilola et al., 2012) uses the properties of the Fuzzy C-Means algorithm and K-means algorithm and it is faster and more accurate than other two algorithms. But the combination of two algorithms results in computational complexity. The analysis of Spatial Fuzzy C-Means Clustering, Fuzzy C-Means Clustering and $\mathrm{K}$-means clustering are discussed in Fig. 3-5 respectively. In Spatial Fuzzy C-Means Clustering method and Fuzzy C-Means Clustering, the analysis are based on the objective function and the number of iterations. The membership value of the Spatial Fuzzy C-Means Clustering and its objective function is less than Fuzzy C-Means Clustering. The $\mathrm{K}$-means clustering algorithm gives the analysis based on the cluster centroid, so the pixels are related to only one class. The Modified Spatial Fuzzy C-Means Clustering gives a better result when compared to the existing algorithms because the input image is analyzed based on the rotation of the image with various angles. This rotation gives the cluster boundary of an image and also decides the data pixels inside and outside the clusters. The data pixels that occur outside the boundary will lead the glaucoma, so the proposed algorithm is more efficient than existing algorithms for identifying the glaucoma.

\section{SPATIAL ANALYSIS OF GLAUCOMA DETECTION}

\subsection{Centroid Based Analysis of the Spatial Glaucoma Detection}

The Modified Spatial Fuzzy C Means clustering algorithm is used to detect the glaucoma based on the spatial parameters with angle of rotation. The glaucoma image is processed and clustered using the proposed method in which the image is rotated at various angles.

The objective of the proposed method focuses on obtaining the different data points with centroid and mean value by rotating an input image in some angle. The clusters are formed based on the distance calculated over the image after rotation. The optic cup size of the input image is compared with original image by measuring the distance from normal to extended size. This spatial rotation is mainly to identify the cluster centroid in order to detect the glaucoma with maximum level. For example the 
centriod value for the 30 degree is $146.1079,161.5851$ which is compared over the normal centroid value for the $30 \mathrm{~m}$ degree is $143.1809,158.5085$. The centroids with clusters are shown in the Fig. 6.

The glaucoma detection is calculated based on various spatial parameters over the fundus images are described in the following section. The input image and original image are represented by the $\mathrm{NxN}$ matrix i.e., array of data elements. Here the $I_{1}$ is an input image and $\mathrm{I}_{2}$ is an original image. The proposed work is used to determine whether the input image has glaucoma or not.

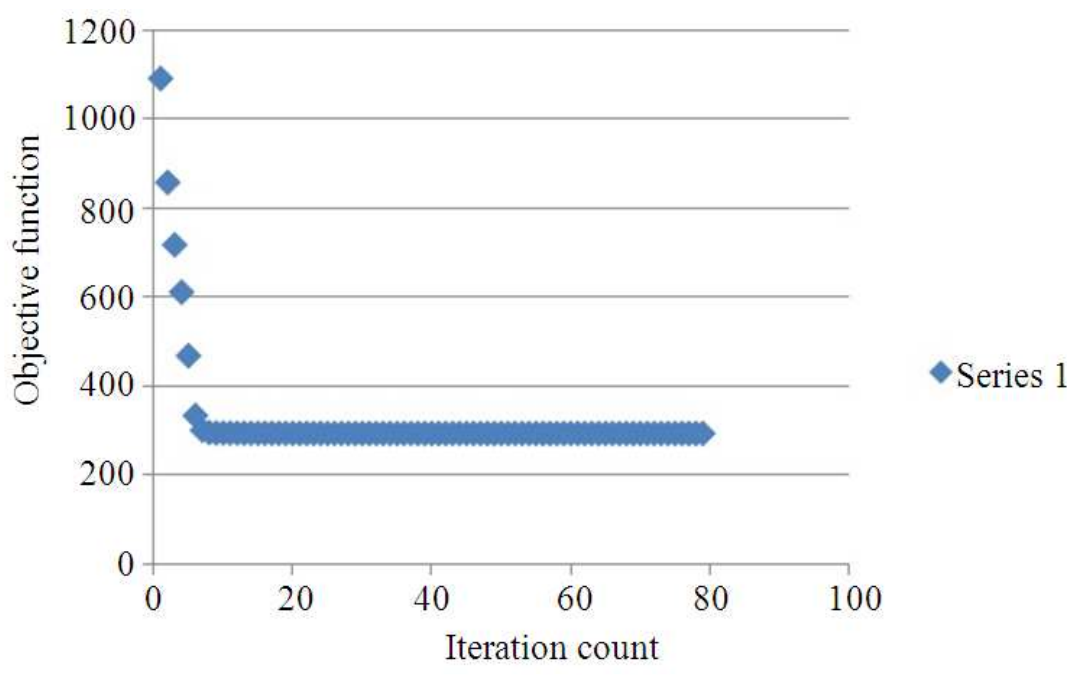

Fig. 3. Spatial fuzzy C-means clustering

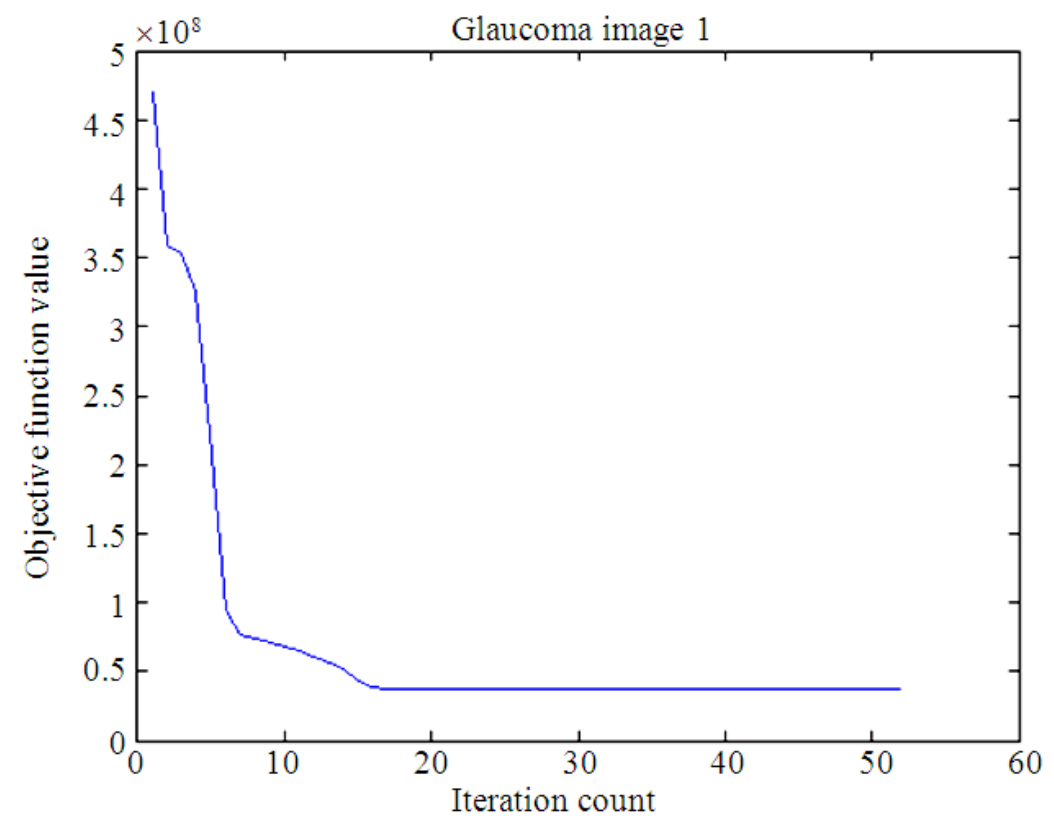

Fig. 4. Fuzzy C-Means clusteing 


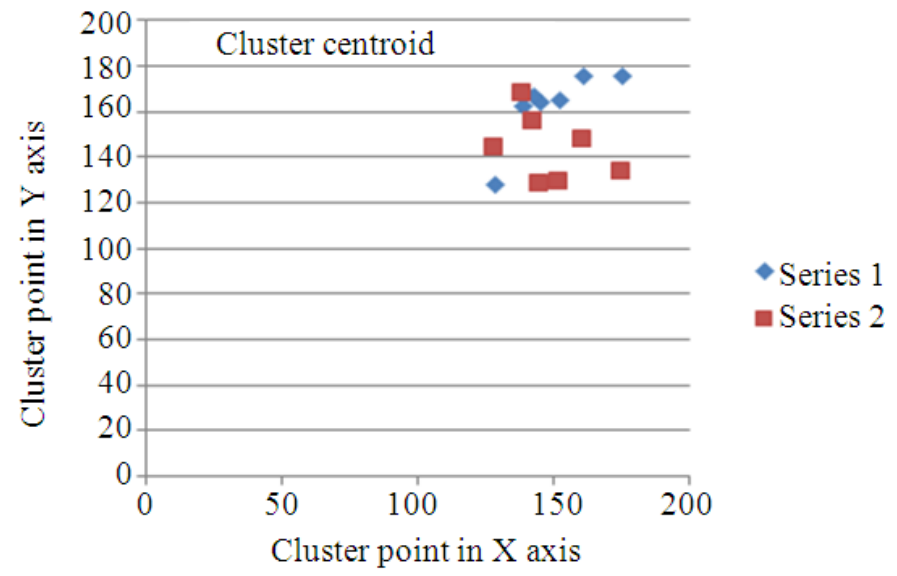

Fig. 5. K-Means clustering

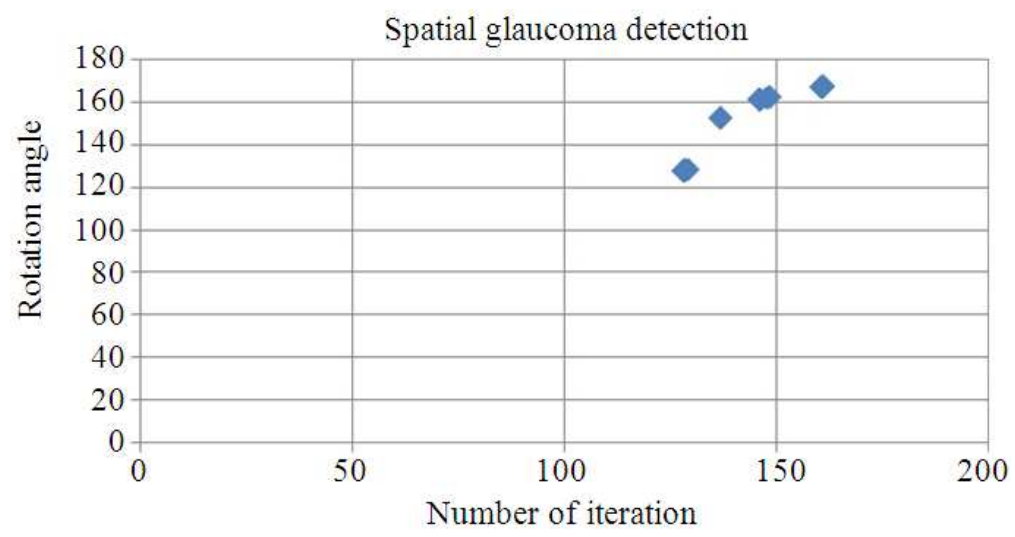

Fig. 6. Analysis of the spatial glaucoma detection

The Modified Spatial Fuzzy C-Means Clustering algorithm compares two images which belong to same cluster with membership value in different angles of rotation. Integrating a spatial fuzzy clustering for automated medical image segmentation is discussed in (Li et al., 2011).

\subsection{Mathematical Analysis of Spatial Glaucoma Detection}

$I_{1}=\left[\begin{array}{cccc}r_{11} & r_{12} & \cdot & r_{1 n} \\ r_{21} & r_{22} & \cdot & r_{2 n} \\ \cdot & \cdot & \cdot & \cdot \\ r_{m 1} & r_{m 2} & \cdot & r_{m n}\end{array}\right]$

(1)

$$
\mathrm{I}_{2}=\left[\begin{array}{cccc}
\mathrm{r}_{11} & \mathrm{r}_{12} & \cdot & \mathrm{r}_{1 \mathrm{n}} \\
\mathrm{r}_{21} & \mathrm{r}_{22} & \cdot & \mathrm{r}_{2 \mathrm{n}} \\
\cdot & \cdot & \cdot & \cdot \\
\mathrm{r}_{\mathrm{m} 1} & \mathrm{r}_{\mathrm{m} 2} & \cdot & \mathrm{r}_{\mathrm{mn}}
\end{array}\right]
$$

The images are rotated in various angle then the outliers are easily identified from the input image Equation 1-4:

$$
I_{1}=\theta\left[I_{1}\right]
$$

$$
\mathrm{I}_{2}=\theta\left[\mathrm{I}_{2}\right]
$$


The RGB Image is converted to gray scale image for further processing by using Equation 5-6:

$$
\begin{gathered}
\mathrm{I}_{1}=\mathrm{X}\left[\theta\left[\mathrm{I}_{1}\right]\right] \\
\mathrm{I}_{2}=\mathrm{X}\left[\theta\left[\mathrm{I}_{2}\right]\right]
\end{gathered}
$$

The noise is removed by using filter for efficient processing of the image is discussed in the Equation 7 and 8:

$$
\begin{aligned}
& I_{1}=\text { Filter }\left[X\left[\theta\left[I_{1}\right]\right]\right] \\
& I_{2}=\text { Filter }\left[X\left[\theta\left[I_{2}\right]\right]\right]
\end{aligned}
$$

The center of the image with respective angle of rotation is calculated in Equation 9-10:

$$
\begin{aligned}
& \text { Center_Input_Image } \\
& =M_{-} F^{*} \text { Input_Image_Data / (Data } * \sum_{i=1}^{\text {max_itr }} M_{-} \text {F) }
\end{aligned}
$$

Center_Original_Image $=$

M_F*Original_Image_Data / (Data $\left.* \sum_{\mathrm{i}=1}^{\text {max_itr }} \mathrm{M}_{-} \mathrm{F}\right)$

The objective function related to the rotation is determined in Equation 11 and 12:
Objective_function_Input_image

$=\sum\left(\sum(\text { Dis } \tan c e)^{2} * M_{-}\right.$F $)$

Objective_function_Original_image

$=\sum\left(\sum(\text { Dis } \tan c e)^{2} * M_{-}\right.$F $)$

The image comparison and final detection of glaucoma is carried out using the Equation 13-14:

$$
\begin{aligned}
& \text { Image_Comparision }= \\
& \left\{\begin{array}{cc}
\text { True, } & \text { if }((\mathrm{I} 1 \cup \mathrm{MF} \cup \mathrm{C} \cup 0 \mathrm{~F}) \\
\text { False } & \cap(\mathrm{I} 2 \cup \mathrm{MF} \cup \mathrm{C} \cup 0 \mathrm{~F}))=\varnothing \\
\text { otherwise }
\end{array}\right.
\end{aligned}
$$

\subsection{Result of the Proposed Algorithm}

Figure 7 and 8 shows that the clustering of original image with various rotations. Figure 9 and 10 shows that the clustering of input image with various rotations. Figure 11 shows that the comparison of original and input image for detecting glaucoma in the retinal fundus images. These figures result in better understanding of detection of glaucoma based on the rotation of optic disk with various angles and also provides a complete result which are not identified in the existing algorithms Equation 14:

$$
\begin{aligned}
& \text { Re sult } \\
& =\left\{\begin{array}{c}
\text { Im age }- \text { Comparision }=\text { true }, \text { No Glaucoma } \\
\text { Im age }- \text { Comparision }=\text { flase }, \text { Glaucoma }
\end{array}\right\}
\end{aligned}
$$

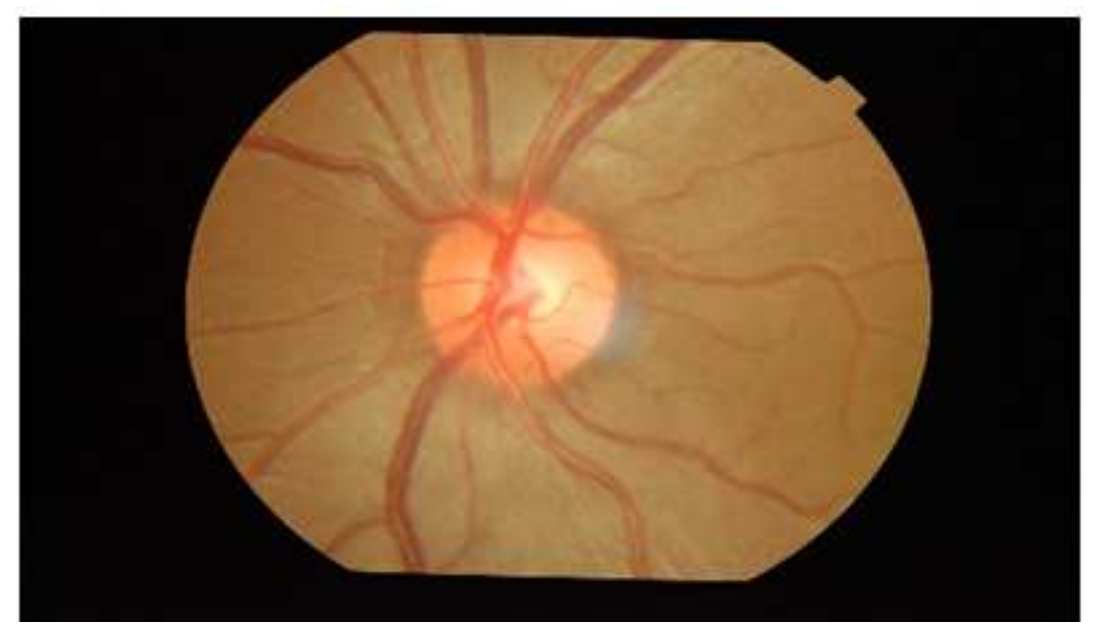

Fig. 7. Original image with clustering 


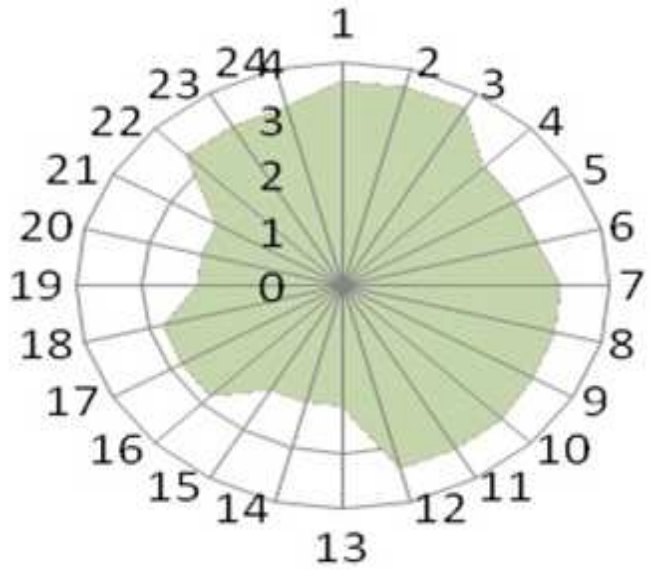

Series 1

Series2

- Series3

Series 4

- Series5

Series 6

Series 7

Fig. 8. Various rotated original image with clustering

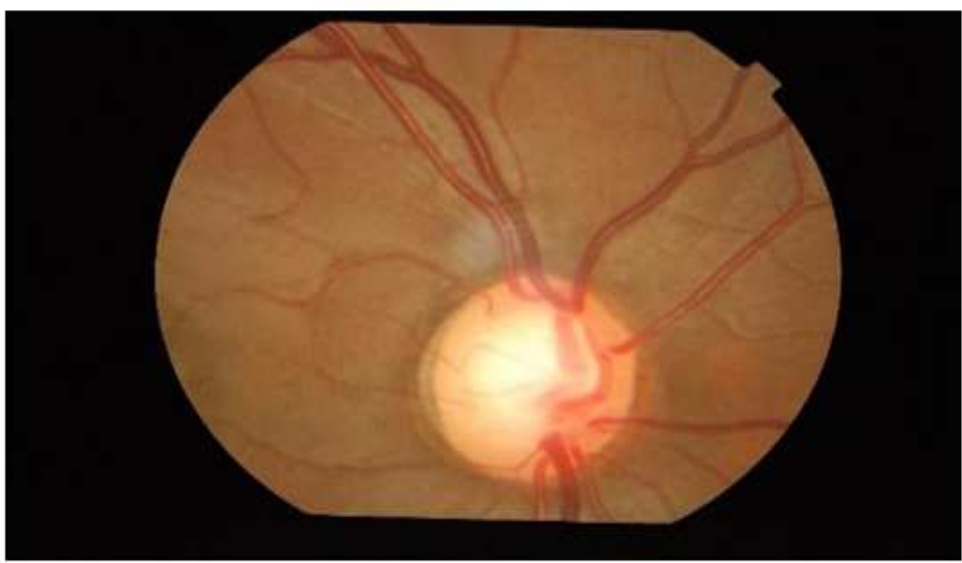

Fig. 9. Input image with clustering

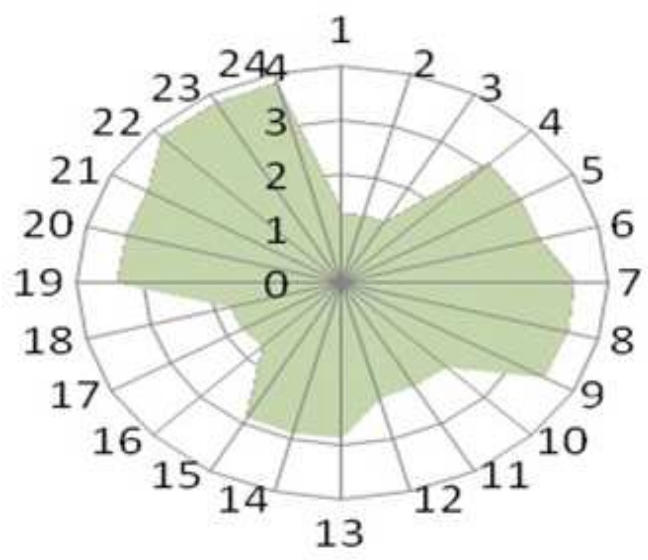

- Series1

- Series2

- Series3

- Series4

- Series5

므 Series6

- Series7

Fig. 10. Various rotated input image with clustering 


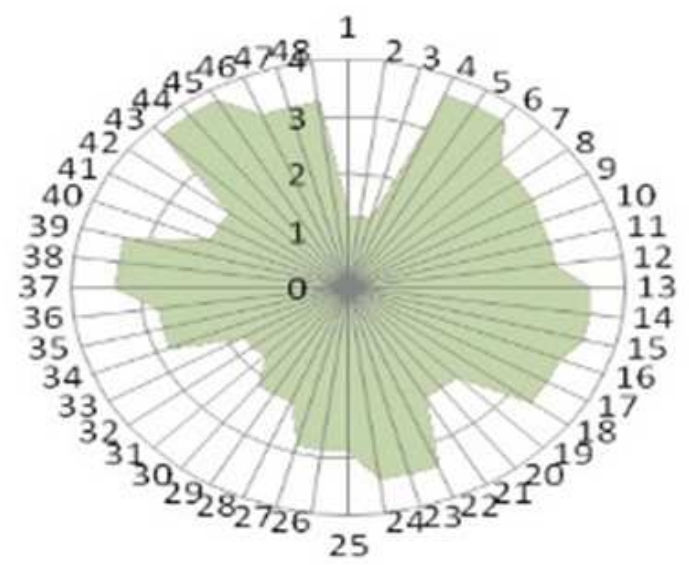

Series1

Series 2

a Series 3

Series 4

Series5

- Series 6

- Series7

- Series 8

= Series 9

Fig. 11. Glaucoma detection using image comparison with clustering

\section{CONCLUSION}

The image analysis and segmentation techniques are used to identify the glaucoma in the human eye. There are two types of learning techniques implemented to detect the glaucoma in the medical image. The threshold technique is used to classify the pixel intensities in two classes and is not suitable for spatial image processing. The $\mathrm{K}$-means clustering technique is analyzed and also compared with the Fuzzy C-Means clustering technique. The Fuzzy K-C-Means clustering is faster and more accurate when compared to these techniques but it is complex. A Modified Spatial Fuzzy C-Means clustering is proposed to overcome the issues related to the existing techniques. The process of the proposed algorithm is to determine the cluster centroid which is calculated based on the distance between the data points of the rotated image. The cluster is formed until no more data points exists in the image. The glaucoma disease in the human eye is identified from the cluster with the occurrence of more data points.This algorithm is restricted to small databases which is related to the glaucoma. In future, the Modified Spatial Fuzzy C Means Clustering algorithm can be extended to larger clinical databases in order to identify the glaucoma at the maximum level.

\section{ACKNOWLEDGMENT}

The author would like to thank egmore Eye Hospital for providing the retinal images to test the proposed method.

\section{REFERENCES}

Anjana, D., K.A. Nair, N. Somashekara, M. Venkata and R. Sripathy et al., 2012. Development of curcumin based ophthalmic formulation. Am. J. Infect. Dis., 8: 41-49. DOI: 10.3844/ajidsp.2012.41.49

Funmilola, A., O.A. Oke, T.O. Adedeji, O.M. Alade and E.A. Adewusi, 2012. Fuzzy K-C-means clustering algorithms for medical image segmentation. J. Inform. Eng. Appli., 2: 21-32.

Kose, C., U. Sevik and O. Gençalioglu, 2012. Simple methods for segmentation and measurement of diabetic retinopathy lesions in retinal fundus images. J. Comput. Methods Programs Biomedicine, 107: 274-293. DOI: 10.1016/j.cmpb.2011.06.007

Kovacs, L., R.J. Qureshi, B. Nagy, B. Harangi and A. Hajdu, 2010. Graph based detection of optic disc and fovea in retinal images. Proceedings of the 4th International Workshop on Soft Computing Applications, Jul. 15-17, IEEE Xplore Press, Arad, pp: 143-148. DOI: 10.1109/SOFA.2010.5565610

Lee, J., B.C.Y. Zee and Q. Li, 2013. Detection of neovascularization based on fractal and texture analysis with interaction effects in diabetic retinopathy. PLoS One, 8: e75699-e75699. DOI: 10.1371/journal.pone.0075699

Li, B.N., C.K. Chui, S. Chang and S.H. Ong, 2011. Integrating spatial fuzzy clustering with level set methods for automated medical image segmentation. Comput. Biol. Medicine, 41: 1-10. DOI: 10.1016/j.compbiomed.2010.10.007 
Mary, M.C.V.S. and B.J.S. Marri, 2012. Automatic optic nerve head segmentation for glaucomatous detection using hough transform and pyramidal decomposition. Int. J. Comput. Applic.

Narasimhan, K. and K. Vijayarekha, 2012. An efficient automated system for glaucoma detection using fundus image. J. Theoretical Applied Inform. Technol., 33: 104-110.

Pinao, J.M.N., 2011. Fovea and optic disk detection and key performance indicators process automation. University of Coimbra.

Raed, T.A. and W. Ashour, 2013. DIMK-means distance-based initialization method for K-means clustering algorithm. Int. J. Intell. Syst. Applic., 5: 41-51. DOI: $10.5815 /$ ijisa.2013.02.05
Welfer, D., J. Scharcanski, C.M. Kitamura, M.M.D. Pizzol and L.W.B. Ludwig et al., 2010. Segmentation of the optic disk in color eye fundus images using an adaptive morphological approach. Comput. Biol. Med., 40: 124-137. DOI: 10.1016/j.compbiomed.2009.11.009

Xiayu, X., 2010. Simultaneous automatic detection of optic disc and fovea. University of Iowa.

Yahya, A.A., J. Tan and M. Hu, 2013. A Novel model of image segmentation based on watershed algorithm. Adv. Multimedia, 2013: 8-8. DOI: $10.1155 / 2013 / 120798$ 